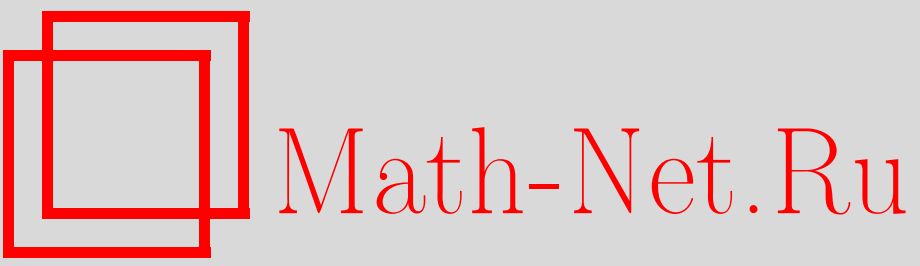

С. Н. Асхабов, Градиентный метод решения нелинейных дискретных и интегральных уравнений с разностными ядрами, Итоги науки и техн. Сер. Соврем. мат. и ее прил. Темат. обз., 2021, том 192, 26-37

DOI: https://doi.org/10.36535/0233-6723-2021-192-26-37

Использование Общероссийского математического портала Math-Net.Ru подразумевает, что вы прочитали и согласны с пользовательским соглашением

http://www.mathnet.ru/rus/agreement

Параметры загрузки:

IP: 54.80 .97 .219

26 апреля 2023 г., 13:48:36 


\title{
ГРАДИЕНТНЫЙ МЕТОД РЕШЕНИЯ НЕЛИНЕЙНЫХ ДИСКРЕТНЫХ И ИНТЕГРАЛЬНЫХ УРАВНЕНИЙ С РАЗНОСТНЫМИ ЯДРАМИ
}

\author{
(c) 2021 г. $\quad$ C. H. ACXАБОВ
}

\begin{abstract}
АннотАция. Методом потенциальных монотонных (по Браудеру-Минти) операторов доказаны глобальные теоремы о существовании и единственности решения для дискретных и интегральных уравнений с разностными ядрами и нечетностепенными нелинейностями. В соответствии с градиентным методом (или методом наискорейшего спуска) построены последовательные приближения, которые сходятся к этим решениям по норме рассматриваемых пространств.
\end{abstract}

Ключевые слова: монотонный оператор, потенциальный оператор, нелинейное дискретное уравнение, нелинейное интегральное уравнение.

\section{GRADIENT METHOD FOR SOLVING NONLINEAR DISCRETE AND INTEGRAL EQUATIONS WITH DIFFERENCE KERNELS}

\author{
(c) 2021 S. N. ASKHABOV
}

\begin{abstract}
The method of potential monotonic operators (called also the Browder-Minty method) is used to prove global theorems on the existence and uniqueness of solutions for discrete and integral equations with difference kernels and odd-power nonlinearities. Using the gradient method (or the steepest descent method), we construct successive approximations that converge to the solutions mentioned with respect to the norm.
\end{abstract}

Keywords and phrases: monotonic operator, potential operator, nonlinear discrete equation, nonlinear integral equation.

AMS Subject Classification: 45G10, 47J05

1. Введение. В работе рассматриваются нелинейные дискретные и интегральные уравнения с разностными ядрами. Для таких уравнений хорошо известны теоремы о существовании и единственности решения в различных (см., например, монографию [1] и приведенную в ней библиографию) пространствах, однако вопрос о том, как найти эти решения, мало изучен. В $[2,4,6]$ комбинированием метода монотонных операторов и принципа сжимающих отображений показано, что решения дискретных и интегральных уравнений типа свертки с монотонной нелинейностью могут быть найдены методом последовательных приближений пикаровского типа в случаях гильбертовых пространств $l_{p}$ и $L_{p}$, соответственно. При этом предполагается, что нелинейности рассматриваемых уравнений удовлетворяют условию Липшица, что не позволяет охватить степенные

Работа выполнена при поддержке Российского фонда фундаментальных исследований (проект № 18-41-200001). 
нелинейности. Наша цель доказать, что в случае нечетностепенных нелинейностей решения дискретных и интегральных уравнений с разностными ядрами могут быть найдены градиентным методом.

Для удобства ссылок приведем определения и вспомогательные утверждения, используемые в данной работе, придерживаясь терминологии и обозначений, принятых в монографии [8].

Пусть $X$ - вещественное банахово пространство и $X^{*}$ сопряженное с ним пространство. Обозначим через $\langle y, x\rangle$ значение линейного непрерывного функционала $y \in X^{*}$ на элементе $x \in X$, а через $\|\cdot\|$ и $\|\cdot\|_{*}$ нормы в $X$ и $X^{*}$, соответственно.

Пусть $u, v \in X$. Оператор $A: X \rightarrow X^{*}$ (т.е. действующий из $X$ в $X^{*}$ ) называется

(1) монотонным, если $\langle A u-A v, u-v\rangle \geqslant 0$;

(2) равномерно монотонным, если $\langle A u-A v, u-v\rangle \geqslant \beta(\|u-v\|)$, где $\beta$ - возрастающая на $[0, \infty)$ функция такая, что $\beta(0)=0$;

(3) коэриитивным, если $\langle A u, u\rangle \geqslant \gamma(\|u\|) \cdot\|u\|$, где $\gamma(s)$ - такая вещественная функция неотрицательного аргумента, что $\gamma(s) \rightarrow \infty$ при $s \rightarrow \infty$;

(4) ограниченно липшиц-непрерывным, если $\|A u-A v\|_{*} \leqslant \mu(r) \cdot\|u-v\|$, где $\mu$ - неотрицательная возрастающая на $[0, \infty)$ функция, а $r=\max (\|u\|,\|v\|)$;

(5) хеминепрерывным, если вещественная функция $s \rightarrow\langle A(u+s \cdot v), w\rangle$ непрерывна на $[0,1]$ при любых фиксированных $u, v, w \in X$.

Если $A$ - линейный оператор, то определение монотонного оператора совпадает с определением положительного оператора.

Функционал $f: X \rightarrow \mathbb{R}$ называется дифференцируемым по Гато, если существует такой оператор $A: X \rightarrow X^{*}$, что для всех $u, v \in X$ выполняется равенство

$$
\lim _{t \rightarrow 0} \frac{f(u+t \cdot v)-f(u)}{t}=\langle A u, v\rangle .
$$

При этом оператор $A$ называют градиентом функционала $f$ и пишут $A=\operatorname{grad} f$.

Оператор $A: X \rightarrow X^{*}$ называется потенциальным, если существует такой функционал $f: X \rightarrow \mathbb{R}$, что оператор $A$ является его градиентом. При этом функционал $f$ называют noтенииалом оператора $A$.

Пример 1. Пусть $X$-вещественное рефлексивное банахово пространство и $A: X \rightarrow X^{*}-$ линейный ограниченный симметрический оператор, т.е. $\langle A u, v\rangle=\langle u, A v\rangle$ для всех $u, v \in X$. Тогда $A$ является потенциальным оператором, а его потенциал равен $f(u)=\langle A u, u\rangle / 2$ (см. $[1, \S 1])$.

Банахово пространство $X$ называется строго выпуклым, если для всех $u, v \in X$ из условий $u \neq v,\|u\| \leqslant 1,\|v\| \leqslant 1$ вытекает неравенство $\|u+v\|<2$.

Оператор $J: X \rightarrow X^{*}$, где $X^{*}$ - строго выпуклое пространство, называется дуализующим отображением, если для всех $u \in X$ выполняются равенства $\langle J u, u\rangle=\|u\|^{2}=\|J u\|_{*}^{2}$.

Заметим, что строгая выпуклость сопряженного пространства $X^{*}$ обеспечивает единственность дуализующего отображения $J: X \rightarrow X^{*}$ (см. [7, лемма 21.1]).

Следующая теорема является следствием общего результата, доказанного в [8, теорема 4.2].

Теорема 1. Пусть $X$ - вещественное рефлексивное банахово пространство и $A: X \rightarrow X^{*}-$ хеминепрерывный равномерно монотонный коэриитивный оператор. Тогда уравнение $А и=f$ имеет единственное решение $u^{*} \in X$ при любом $f \in X^{*}$. Кроме того, если $X$ и $X^{*}$ строго выпуклые пространства, а оператор $A$ является потенииальным ограниченно липшиц-непрерывным, то последовательность

$$
u_{n+1}=u_{n}-\delta_{n} \cdot J^{*}\left(A u_{n}-f\right),
$$

¿əe

$$
\delta_{n}=\min \left\{1,2 /\left[\varepsilon+\mu\left(\left\|u_{n}\right\|+\left\|A u_{n}-f\right\|_{*}\right)\right]\right\}, \quad n=0,1,2,3, \ldots,
$$

$J^{*}: X^{*} \rightarrow X-$ дуализующее отображсние для $X^{*}, \varepsilon>0-$ произвольное число, сходится $\kappa u^{*}$ по норме пространства $X$. 
Доказательство. Существование и единственность решения $u^{*}$ вытекает из теоремы БраудераМинти (см. [8, теорема 2.1]), а сильная сходимость последовательности $\left\{u_{n}\right\}$ к $u^{*}$ по указанной схеме - из [8, теорема 4.2, замечание 4.13], поскольку всякий равномерно монотонный оператор является строго монотонным оператором и обладает (S)-свойством (см. [8, замечание 1.5]).

Указанный в теореме 1 способ нахождения решения $u^{*}$ известен как градиентныц метод (или метод наискорейшего спуска), так как $J^{*} v=\|v\|_{*} \cdot \operatorname{grad}\|v\|_{*}$ для всех $v \in X^{*}$ (см. [8]).

Всюду далее через $\mathbb{R}, \mathbb{N}, \mathbb{Z}$ и $\mathbb{C}$ обозначаются, соответственно, множества всех действительных, натуральных, целых и комплексных чисел, а через $p^{\prime}$ - сопряженное с $p>1$ число: $p^{\prime}=p /(p-1)$.

\section{2. Нелинейные дискретные уравнения типа свертки.}

2.1. Дискретные уравнения в весовом вещественном пространстве $\ell_{p}(\varrho)$. Рассмотрим дискретный оператор свертки $H$ :

$$
(H u)_{n}=(h * u)_{n}=\sum_{k=-\infty}^{\infty} h_{n-k} u_{k}, \quad n \in \mathbb{Z},
$$

в вещественных пространствах числовых последовательностей $\ell_{p}, 1 \leqslant p \leqslant \infty$, с нормой

$$
\|u\|_{p}=\left(\sum_{n=-\infty}^{\infty}\left|u_{n}\right|^{p}\right)^{1 / p} \text { при } 1 \leqslant p<\infty, \quad\|u\|_{\infty}=\sup _{n \in \mathbf{Z}}\left|u_{n}\right| \text { при } p=\infty .
$$

Теорема 2. Пусть $1 \leqslant p \leqslant 2$ и ядро $h \in \ell_{s}, 1 \leqslant s \leqslant \min \left(2, p^{\prime} / 2\right)$. Для того, чтобы дискретный оператор свертки $Н$ был положительным в пространстве $\ell_{p}$ необходимо и достаточно, чтобы выполнялось условие

$$
\widehat{h}_{c}(\theta)=\sum_{k=-\infty}^{\infty} h_{k} \cdot \cos (k \cdot \theta) \geqslant 0, \quad \theta \in[0, \pi] .
$$

Заметим, что при условиях теоремы 2 оператор $H$ действует непрерывно из $\ell_{p}$ в $\ell_{p^{\prime}}$, причем $\|H u\|_{p^{\prime}} \leqslant\|h\|_{s} \cdot\|u\|_{p}$ (см. [1, следствие 29.1]).

Широкий класс ядер, удовлетворяющих условию (2) предоставляет следующий пример.

Пример 2 (см. [2]). Если ядро $h=\left\{h_{n}\right\}_{n=-\infty}^{\infty}$ представимо в виде

$$
h_{n}=\sum_{k=-\infty}^{\infty} g_{n+k} \cdot g_{k}, \quad \text { где } g=\left\{g_{n}\right\}_{n=-\infty}^{\infty} \in \ell_{1},
$$

то $\widehat{h}_{c}(\theta) \geqslant 0$ при всех $\theta \in[0, \pi]$.

Лемма 1. Пусть $1 \leqslant p \leqslant \infty$ и ядро $h=\left\{h_{n}\right\}_{n=-\infty}^{\infty}$ представимо в виде (3). Тогда $h \in \ell_{p}$ и обладает следующими свойствами: $h_{-n}=h_{n}$ при всех $n \in \mathbb{Z} u\|h\|_{p} \leqslant\|g\|_{1} \cdot\|g\|_{p}$.

Введем в рассмотрение весовое пространство $\ell_{p}(\varrho)$. Пусть $\varrho=\left\{\varrho_{n}\right\}_{n=-\infty}^{\infty}$ есть последовательность положительных чисел. Обозначим через $\ell_{p}(\varrho), 1<p<\infty$, множество всех вещественных числовых последовательностей $u=\left\{u_{n}\right\}_{n=-\infty}^{\infty}$ таких, что

$$
\|u\|_{p, 1}=\left(\sum_{n=-\infty}^{\infty} \varrho_{n}\left|u_{n}\right|^{p}\right)^{1 / p}<\infty
$$

Известно, что $\ell_{p}(\varrho)$ является рефлексивным банаховым пространством и $\ell_{p}^{*}(\varrho)=\ell_{p^{\prime}}\left(\varrho^{1-p^{\prime}}\right)$, $p^{\prime}=p /(p-1)$, есть сопряженное с ним пространство. Норму в $\ell_{p^{\prime}}\left(\varrho^{1-p^{\prime}}\right)$ обозначим через $\|\cdot\|_{p^{\prime}, 1-p^{\prime}}$.

Известно, что для пространств $\ell_{p}$ имеет место непрерывное вложение $\ell_{p} \subset \ell_{q}$, если $p<q$, в отличие от пространств Лебега $L_{p}(a, b)$ для которых наоборот $L_{q}(a, b) \subset L_{p}(a, b)$, если $p<q$. Оказывается, что в случае весовых пространств $\ell_{p}(\varrho)$, в отличие от весовых пространств $L_{p}(\varrho)$, возможно обратное вложение: $\ell_{q}\left(\varrho_{1}\right) \subset \ell_{p}\left(\varrho_{2}\right)$, если $p<q$. А именно, справедливо следующее утверждение. 
Лемма 2. Пусть $2 \leqslant p<\infty$ иес $\varrho=\left\{\varrho_{n}\right\}_{n=-\infty}^{\infty}$ удовлетворяет условию

$$
\gamma=\left(\sum_{n=-\infty}^{\infty} \varrho_{n}^{-2 /(p-2)}\right)^{(p-2) /(2 p)}<\infty
$$

$\left(\right.$ при $p=2$ полагаем $\left.\gamma=\sup _{n \in \mathbb{Z}} \varrho_{n}^{-1 / 2}\right)$. Тогда справедливы непрерывные вложения

$$
\ell_{p}(\varrho) \subset \ell_{2} \subset \ell_{p^{\prime}}\left(\varrho^{1-p^{\prime}}\right)
$$

причем

$$
\|u\|_{2} \leqslant \gamma \cdot\|u\|_{p, 1}, \quad u \in \ell_{p}(\varrho), \quad\|u\|_{p^{\prime}, 1-p^{\prime}} \leqslant \gamma \cdot\|u\|_{2}, \quad u \in \ell_{2}
$$

При исследовании нелинейного дискретного уравнения типа свертки в весовом пространстве $\ell_{p}(\varrho)$ существенно будет использоваться следующая лемма.

Лемма 3. Пусть $2 \leqslant p<\infty$, ядро $h$ представимо в виде (3) и вес $\varrho=\left\{\varrho_{n}\right\}_{n=-\infty}^{\infty}$ yдовлетворяет условию (4). Тогда дискретный оператор свертки H, определенный равенством (1), действует непрерывно из $\ell_{p}(\varrho)$ в $\ell_{p^{\prime}}\left(\varrho^{1-p^{\prime}}\right)$, положителен и потенииален, причем

$$
\|H u\|_{p^{\prime}, 1-p^{\prime}} \leqslant \gamma^{2} \cdot\|g\|_{1}^{2} \cdot\|u\|_{p, 1}, \quad u \in \ell_{p}(\varrho) .
$$

Доказательство лемм 1- 3 приведено в [2].

Из неравенств Кларксона для весовых пространств $\ell_{p}(\varrho)$ (см. [2]) непосредственно вытекает, что $\ell_{p}(\varrho)$ и $\ell_{p^{\prime}}\left(\varrho^{1-p^{\prime}}\right)$ при $1<p<\infty$ являются строго выпуклыми пространствами, причем для пространств $\ell_{p}(\varrho), 1<p<\infty$, дуализующее отображение однозначно определяется по формуле $J u=\|u\|_{p, 1}^{2-p} \cdot z$, где $z=\left\{\varrho_{n}\left|u_{n}\right|^{p-2} u_{n}\right\}_{n=-\infty}^{\infty}($ случай $\varrho=1$ см. в [7]).

Заметим, что дуализующее отображение $J^{*}$ для сопряженного пространства $\ell_{p^{\prime}}\left(\varrho^{1-p^{\prime}}\right)$ единственным образом определяется по формуле:

$$
J^{*} v=\|v\|_{p^{\prime}, 1-p^{\prime}}^{2-p^{\prime}} \cdot z, \text { где } z=\left\{\varrho_{n}^{1-p^{\prime}}\left|v_{n}\right|^{p^{\prime}-2} v_{n}\right\}_{n=-\infty}^{\infty} .
$$

Непосредственно проверяется, что $\left\langle J^{*} v, v\right\rangle=\|v\|_{p^{\prime}, 1-p^{\prime}}^{2}=\left\|J^{*} v\right\|_{p, 1}^{2}$ при всех $v \in \ell_{p^{\prime}}\left(\varrho^{1-p^{\prime}}\right)$.

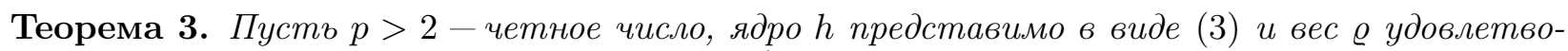
ряет условию (4). Тогда при любом $f \in \ell_{p^{\prime}}\left(\varrho^{1-p^{\prime}}\right)$ нелинейное дискретное уравнение

$$
\varrho_{n} \cdot u_{n}^{p-1}+\sum_{k=-\infty}^{\infty} h_{n-k} u_{k}=f_{n}, \quad n \in \mathbb{Z}
$$

имеет единственное решение $u^{*} \in \ell_{p}(\varrho)$. K этому решению сходится по норме пространства $\ell_{p}(\varrho)$ последовательность $u^{(i)}=\left\{u_{n}^{(i)}\right\}_{n=-\infty}^{\infty}$, определяемая по бормуле

$$
u_{n}^{(i+1)}=u_{n}^{(i)}-\delta^{(i)} \cdot \varrho_{n}^{-1 /(p-1)} \cdot\left\|A u^{(i)}-f\right\|_{p^{\prime}, 1-p^{\prime}}^{(p-2) /(p-1)} \cdot\left|A u_{n}^{(i)}-f_{n}\right|^{(2-p) /(p-1)} \cdot\left(A u_{n}^{(i)}-f_{n}\right),
$$

где $i=0,1,2, \ldots, u^{(0)}=\left\{u_{n}^{(0)}\right\}_{n=-\infty}^{\infty} \in \ell_{p}(\varrho)-$ любое начальное приближение, $A u=\varrho \cdot u^{p-1}+H u$,

$$
\delta^{(i)}=\min \left\{1, \frac{2}{\varepsilon+(p-1) \cdot\left(\left\|u^{(i)}\right\|_{p, 1}+\left\|A u^{(i)}-f\right\|_{p^{\prime}, 1-p^{\prime}}\right)^{p-2}+\gamma^{2}\|g\|_{1}^{2}}\right\}, \quad \varepsilon>0 .
$$

Доказательство. Запишем уравнение (9) в операторном виде: $A u=f$, где $A u=\varrho \cdot u^{p-1}+$ $H u$. Так как $\left\|\varrho \cdot u^{p-1}\right\|_{p^{\prime}, 1-p^{\prime}}=\|u\|_{p, 1}^{p-1}$, то оператор $B u=\varrho \cdot u^{p-1}$ действует непрерывно из $\ell_{p}(\varrho)$ в $\ell_{p^{\prime}}\left(\varrho^{1-p^{\prime}}\right)$ и потенциален (см. [8, лемма 4.1]). Поэтому оператор $A$, будучи суммой двух непрерывных потенциальных операторов, является непрерывным потенциальным оператором, действующим из $\ell_{p}(\varrho)$ в $\ell_{p^{\prime}}\left(\varrho^{1-p^{\prime}}\right)$. 
Покажем, что оператор $A$ является равномерно монотонным. Используя лемму 3 и неравенство $\left(t^{p-1}-s^{p-1}\right)(t-s) \geqslant 2^{2-p}|t-s|^{p}$, справедливое для всех $t, s \in \mathbb{R}$ и четных $p \geqslant 2$, имеем

$$
\begin{aligned}
& \langle A u-A v, u-v\rangle=\sum_{n=-\infty}^{\infty} \varrho_{n}\left(u_{n}^{p-1}-v_{n}^{p-1}\right)\left(u_{n}-v_{n}\right)+\langle H(u-v), u-v\rangle \geqslant \\
& \quad \geqslant 2^{2-p} \sum_{n=-\infty}^{\infty} \varrho_{n}\left|u_{n}-v_{n}\right|^{p}=\beta\left(\|u-v\|_{p, 1}\right), \quad u, v \in \ell_{p}(\varrho),
\end{aligned}
$$

где $\beta(x)=2^{2-p} x^{p}$ строго возрастающая на $[0, \infty)$ функция и $\beta(0)=0$. Значит, $A-$ равномерно монотонный оператор, и по теореме 1 уравнение $A u=f$ имеет единственное решение $u^{*} \in \ell_{p}(\varrho)$.

Осталось доказать, что это решение можно найти по итерационной формуле (10). Поскольку пространства $\ell_{p}(\varrho)$ и $\ell_{p^{\prime}}\left(\varrho^{1-p^{\prime}}\right)$ являются строго выпуклыми, и оператор $A$ является непрерывным, равномерно монотонным и потенциальным, то с учетом явного вида (8) дуализующего отображения $J^{*}$ на основании теоремы 1 достаточно доказать, что оператор $A$ является ограниченно липшиц-непрерывным. Используя числовое неравенство

$$
\left|t^{p-1}-s^{p-1}\right| \leqslant \frac{p-1}{2}|t-s|\left(t^{p-2}+s^{p-2}\right),
$$

справедливое для всех $t, s \in \mathbb{R}$ и четных $p \geqslant 2$, для любых $u, v \in \ell_{p}(\varrho)$ имеем

$$
\begin{gathered}
\left\|\varrho u^{p-1}-\varrho v^{p-1}\right\|_{p^{\prime}, 1-p^{\prime}}=\left(\sum_{n=-\infty}^{\infty} \varrho_{n}^{1-p^{\prime}}\left|\varrho_{n}\left(u_{n}^{p-1}-v_{n}^{p-1}\right)\right|^{p^{\prime}}\right)^{\frac{1}{p^{\prime}}}= \\
=\left(\sum_{n=-\infty}^{\infty} \varrho_{n}\left|u_{n}^{p-1}-v_{n}^{p-1}\right|^{p^{\prime}}\right)^{\frac{1}{p^{\prime}}} \leqslant \frac{p-1}{2}\left(\sum_{n=-\infty}^{\infty} \varrho_{n}\left|u_{n}-v_{n}\right|^{p^{\prime}}\left|u_{n}^{p-2}+v_{n}^{p-2}\right|^{p^{\prime}}\right)^{\frac{1}{p^{\prime}}}= \\
=\frac{p-1}{2}\left(\sum_{n=-\infty}^{\infty} \varrho_{n}^{p^{\prime}-1}\left|u_{n}-v_{n}\right|^{p^{\prime}} \varrho_{n}^{2-p^{\prime}}\left|u_{n}^{p-2}+v_{n}^{p-2}\right|^{p^{\prime}}\right)^{\frac{1}{p^{\prime}}} \leqslant
\end{gathered}
$$

(применяем неравенство Гельдера с показателями $p / p^{\prime}$ и $p /\left(p-p^{\prime}\right)$, где $p>2$ четное число)

$$
\begin{gathered}
\leqslant \frac{p-1}{2}\left(\left[\sum_{n=-\infty}^{\infty} \varrho_{n}^{\frac{\left(p^{\prime}-1\right) p}{p^{\prime}}}\left|u_{n}-v_{n}\right|^{p}\right]^{\frac{p^{\prime}}{p}}\left[\sum_{n=-\infty}^{\infty} \varrho_{n}^{\frac{\left(2-p^{\prime}\right) p}{p-p^{\prime}}}\left|u_{n}^{p-2}+v_{n}^{p-2}\right|^{\frac{p p^{\prime}}{p-p^{\prime}}}\right]^{\frac{p-p^{\prime}}{p}}\right)^{\frac{1}{p^{\prime}}}= \\
=\frac{p-1}{2}\|u-v\|_{p, 1}\left(\sum_{n=-\infty}^{\infty} \varrho_{n}\left|u_{n}^{p-2}+v_{n}^{p-2}\right|^{\frac{p}{p-2}}\right)^{\frac{p-2}{p}} \leqslant
\end{gathered}
$$

(вносим $\varrho_{n}$ под знак модуля и затем применяем неравенство Минковского)

$$
\leqslant \frac{p-1}{2}\|u-v\|_{p, 1}\left(\|u\|_{p, 1}^{p-2}+\|v\|_{p, 1}^{p-2}\right) \leqslant(p-1) r^{p-2}\|u-v\|_{p, 1},
$$

где $r=\max \left(\|u\|_{p, 1},\|v\|_{p, 1}\right)$. Используя последнее неравенство и неравенство (7), получаем

$$
\begin{aligned}
\|A u-A v\|_{p, 1} \leqslant\left\|\varrho u^{p-1}-\varrho v^{p-1}\right\|_{p, 1} & +\|H(u-v)\|_{p, 1} \leqslant \\
& \leqslant(p-1) r^{p-2}\|u-v\|_{p, 1}+\gamma^{2}\|g\|_{1}^{2}\|u-v\|_{p, 1}=\mu(r)\|u-v\|_{p, 1},
\end{aligned}
$$

где $\mu(r)=(p-1) r^{p-2}+\gamma^{2}\|g\|_{1}^{2}$ - возрастающая на $[0, \infty)$ функция. Значит, $A$ - ограниченно липшиц-непрерывный оператор.

В связи с теоремой 2 отметим, что в $[1,2,10,14]$ изучены различные классы нелинейных дискретных уравнений и, в частности, дискретные уравнения типа Гаммерштейна. 
2.2. Дискретные операторы свертки в комплексном пространстве $l_{p}$. Аналогичные результаты имеют место в случае комплексных пространств $l_{p}$ и операторов более общего, чем $H$, вида. Обозначим через $l_{p}, 1 \leqslant p \leqslant \infty$, пространство, состоящее из последовательностей комплексных чисел $u=\left\{u_{n}\right\}_{n=-\infty}^{\infty}$ таких, что

$$
\|u\|_{p}^{p}=\sum_{n=-\infty}^{\infty}\left|u_{n}\right|^{p}<\infty \quad \text { при } 1 \leqslant p<\infty, \quad\|u\|_{\infty}=\sup _{n \in \mathbb{Z}}\left|u_{n}\right|<\infty \quad \text { при } p=\infty .
$$

Хорошо известно, что $l_{p}$ при $1<p<\infty$ есть сепарабельное рефлексивное банахово пространство, $l_{p^{\prime}}, p^{\prime}=p /(p-1)$, является сопряженным с ним пространством и общий вид линейного непрерывного функционала в $l_{p}$ определяется по формуле

$$
\langle y, x\rangle=\sum_{n=-\infty}^{\infty} y_{n} \cdot \overline{x_{n}}, \text { где } y \in l_{p^{\prime}}, x \in l_{p},
$$

(черта сверху означает знак комплексного сопряжения), причем $\langle y, x\rangle$ совпадает со скалярным произведением $(y, x)$ в гильбертовом пространстве $l_{2}$, т.е. при $p=p^{\prime}=2$.

Определим в пространстве $l_{2}$ дискретный оператор свертки $H$ с помощью равенства (1). Известно, что если $h \in l_{1}$, то оператор $H$ действует непрерывно из $l_{2}$ в $l_{2}$, причем справедливо дискретное неравенство Юнга $\|H u\|_{2} \leqslant\|h\|_{1} \cdot\|u\|_{2}$.

Обозначим через $\widehat{h}(\Theta)$ и $h(t)$, где $\widehat{h}(\Theta)=h(t)$ при $t=\exp (i \cdot \Theta)$, соответственно, дискретные преобразования Фурье и Лорана последовательности $h=\left\{h_{n}\right\}_{n=-\infty}^{\infty}($ см. $[9, \S 24])$ :

$$
\begin{gathered}
\widehat{h}(\Theta)=\sum_{n=-\infty}^{\infty} h_{n} \cdot \exp (i n \Theta), \quad h_{n}=\frac{1}{2 \pi} \int_{-\pi}^{\pi} \widehat{h}(\Theta) \exp (i n \Theta) d \Theta, \\
h(t)=\sum_{n=-\infty}^{\infty} h_{n} \cdot t^{n}, \quad h_{n}=\frac{1}{2 \pi i} \int_{|t|=1} h(t) \cdot t^{-n-1} d t .
\end{gathered}
$$

Следуя [12, п. 2.2.4], обозначим через $L_{p}(-\pi, \pi), 1 \leqslant p \leqslant \infty$, множество всех измеримых на интервале $(-\pi, \pi)$ комплекснозначных $2 \pi$-периодических функций $f(x)$, удовлетворяющих условиям

$$
\begin{array}{ll}
\|f\|_{L_{p}(-\pi, \pi)}=\left[\frac{1}{2 \pi} \int_{-\pi}^{\pi}|f(x)|^{p} d x\right]^{1 / p}<\infty & \text { при } 1 \leqslant p<\infty, \\
\|f\|_{L_{\infty}(-\pi, \pi)}= & \text { ess } \sup _{-\pi \leqslant x \leqslant \pi}|f(x)|<\infty
\end{array}
$$

Докажем следующую лемму, играющую важную роль при исследовании методом монотонных операторов нелинейных дискретных уравнений типа свертки в комплексных пространствах $l_{p}$.

Лемма 4. Пусть $1<p<\infty$, ядро $h \in l_{1}$ и удовлетворяет условию

$$
\operatorname{Re} \widehat{h}(\Theta)=\operatorname{Re} \sum_{n=-\infty}^{\infty} h_{n} \cdot \exp (i n \Theta) \geqslant 0, \quad \Theta \in(-\pi, \pi)
$$

Если выполнено условие:

$$
b \in l_{\infty} \text { nри } 1<p \leqslant 2 \quad \text { u } \quad b \in l_{2 p /(p-2)} \text { при } 2<p<\infty,
$$

то дискретный оператор свертки $B$, определяемый по правилу

$$
(B u)_{n}=\overline{b_{n}} \sum_{k=-\infty}^{\infty} b_{k} h_{n-k} u_{k}, \quad n \in \mathbb{Z}
$$


действует непрерывно из пространства $l_{p}$ в сопряженное с ним пространство $l_{p^{\prime}}$ и положителен, причем для всех $u \in l_{p}$ справедливы неравенство

$$
\|B u\|_{p^{\prime}} \leqslant C \cdot\|u\|_{p}
$$

гde

и равенство

$$
C= \begin{cases}\|b\|_{\infty}^{2} \cdot\|h\|_{1}, & \text { если } 1<p \leqslant 2 \\ \|b\|_{2 p /(p-2)}^{2} \cdot\|h\|_{1}, & \text { если } 2<p<\infty\end{cases}
$$

$$
\operatorname{Re}\langle B u, u\rangle=\frac{1}{2 \pi} \int_{-\pi}^{\pi} \operatorname{Re} \widehat{h}(\Theta) \cdot|\widehat{b \cdot u}(\Theta)|^{2} d \Theta
$$

Доказательство. Докажем сначала неравенство (14).

1. Пусть $p=2$. Тогда в силу дискретного неравенства Юнга с учетом того, что $\left|\overline{b_{n}}\right|=\left|b_{n}\right|$ и $\|b \cdot u\|_{2} \leqslant\|b\|_{\infty}\|u\|_{2}$, т.е. $b \cdot u \in l_{2}$, имеем

$$
\|B u\|_{2} \leqslant\|\bar{b}\|_{\infty}\left(\sum_{n=-\infty}^{\infty}\left|\sum_{k=-\infty}^{\infty} h_{n-k} b_{k} u_{k}\right|^{2}\right)^{1 / 2} \leqslant\|b\|_{\infty}\|h\|_{1}\|b \cdot u\|_{2} \leqslant\|b\|_{\infty}^{2}\|h\|_{1}\|u\|_{2} .
$$

Таким образом, неравенство (14) доказано при $p=2$ :

2. Пусть $1<p<2$. Тогда $p^{\prime}>2$ и имеют место непрерывные вложения $l_{p} \subset l_{2} \subset l_{p^{\prime}}$, причем справедливы неравенства [12, п. 2.2.5]:

$$
\|u\|_{p^{\prime}} \leqslant\|u\|_{2} \leqslant\|u\|_{p}, \quad u \in l_{p} .
$$

Заметим, что $b \cdot u \in l_{2}$ для всех $u \in l_{p}$ в силу вложения $l_{p} \subset l_{2}$ и очевидного неравенства $\|b \cdot u\|_{2} \leqslant\|b\|_{\infty}\|u\|_{2}$, использованного в пю 1. Далее, используя неравенства (16) и (17), имеем

$$
\|B u\|_{p^{\prime}} \leqslant\|B u\|_{2} \leqslant\|b\|_{\infty}^{2}\|h\|_{1}\|u\|_{2} \leqslant\|b\|_{\infty}^{2}\|h\|_{1}\|u\|_{p} .
$$

Следовательно, неравенство (14) выполняется при $1<p<2$.

3. Пусть, наконец, $2<p<\infty$. Применяя неравенство Гельдера для сумм, имеем

$$
\|b \cdot u\|_{2} \leqslant \sum_{n=-\infty}^{\infty}\left|b_{n}\right|^{2}\left\|u_{n}\right\|^{2} \leqslant\left(\sum_{n=-\infty}^{\infty}\left|b_{n}\right|^{2 p /(p-2)}\right)^{(p-2) / p}\left(\sum_{n=-\infty}^{\infty}\left|u_{n}\right|^{p}\right)^{2 / p}
$$

откуда

$$
\|b \cdot u\|_{2} \leqslant\|b\|_{2 p /(p-2)}\|u\|_{p}, \quad u \in l_{p}
$$

т.е. $b \cdot u \in l_{2}$. Так как ядро $h \in l_{1}$, то на основании дискретного неравенства Юнга имеем $H(b \cdot u) \in$ $l_{2}$, причем в силу предыдущего неравенства

$$
\|H(b \cdot u)\|_{2} \leqslant\|h\|_{1}\|b \cdot u\|_{2} \leqslant\|h\|_{1}\|b\|_{2 p /(p-2)}\|u\|_{p}, \quad u \in l_{p} .
$$

Применяя неравенство Гельдера для сумм с показателями $2 /\left(2-p^{\prime}\right)$ и $2 / p^{\prime}$, имеем

$$
\|\bar{b} \cdot H(b \cdot u)\|_{p^{\prime}}^{p^{\prime}} \leqslant \sum_{n=-\infty}^{\infty}\left|\overline{b_{n}}\right|^{p^{\prime}} \cdot\left|\sum_{k=-\infty}^{\infty} b_{k} h_{n-k} u_{k}\right|^{p^{\prime}} \leqslant\left(\sum_{n=-\infty}^{\infty}\left|\overline{b_{n}}\right|^{2 p^{\prime} /\left(2-p^{\prime}\right)}\right)^{\left(2-p^{\prime}\right) / 2}\|H(b \cdot u)\|_{2}^{p^{\prime}},
$$

откуда с учетом неравенства (18) и того факта, что $2 p^{\prime} /\left(2-p^{\prime}\right)=2 p /(p-2)$ и $\left|\overline{b_{n}}\right|=\left|b_{n}\right|$, получаем

$$
\|B u\|_{p^{\prime}}=\|\bar{b} \cdot H(b \cdot u)\|_{p^{\prime}} \leqslant\|\bar{b}\|_{2 p /(p-2)}\|H(b \cdot u)\|_{2} \leqslant\|b\|_{2 p /(p-2)}^{2}\|h\|_{1}\|u\|_{p}
$$

т.е. неравенство (14) справедливо при $2<p<\infty$.

Таким образом, в пп. 1-3 неравенство (14) доказано для всех $p \in(1, \infty)$ и, значит, оператор $B$ действует непрерывно из $l_{p}$ в $l_{p^{\prime}}$ при любом $p \in(1, \infty)$. Осталось установить положительность 
оператора $B$. Для этого докажем сначала, что оператор свертки $H$ является положительным в пространстве $l_{2}$, т.е. выполняется неравенство

$$
\operatorname{Re}(H u, u)=\operatorname{Re} \sum_{n=-\infty}^{\infty}\left(\sum_{k=-\infty}^{\infty} h_{n-k} u_{k}\right) \overline{u_{n}} \geqslant 0, \quad u \in l_{2} .
$$

Итак, пусть $h \in l_{1}$ и $u=\left\{u_{n}\right\}_{n=-\infty}^{\infty}-$ произвольная финитная последовательность. Положим

$$
c_{n}=\sum_{k=-\infty}^{\infty} h_{n-k} u_{k}, \quad n \in \mathbb{Z}
$$

Тогда (см. [9, пю 24.3]) $c(t)=h(t) \cdot u(t)$ и, значит,

$$
\sum_{k=-\infty}^{\infty} h_{n-k} u_{k}=c_{n}=\frac{1}{2 \pi i} \int_{|t|=1} h(t) \cdot u(t) \cdot t^{-n-1} d t .
$$

Поэтому, с учетом того, что

$$
\bar{u}(t) \cdot t^{-1}=\sum_{n=-\infty}^{\infty} \overline{u_{n}} \cdot t^{-n-1}
$$

и $\widehat{h}(\Theta)=h(t), \widehat{u}(\Theta)=u(t)$ при $t=\exp (i \Theta)$, имеем

$$
\begin{aligned}
(H u, u) & =\sum_{n=-\infty}^{\infty}\left(\sum_{k=-\infty}^{\infty} h_{n-k} u_{k}\right) \overline{u_{n}}= \\
& =\sum_{n=-\infty}^{\infty}\left[\frac{1}{2 \pi i} \int_{|t|=1} h(t) \cdot u(t) \cdot t^{-n-1} d t\right] \overline{u_{n}}= \\
& =\frac{1}{2 \pi i} \int_{|t|=1} h(t) \cdot u(t) \cdot t^{-n-1}\left(\sum_{n=-\infty}^{\infty} \overline{u_{n}} \cdot t^{-n-1}\right) d t= \\
& =\frac{1}{2 \pi i} \int_{|t|=1} h(t) \cdot u(t) \cdot \bar{u}(t) \cdot t^{-1} d t= \\
& =\frac{1}{2 \pi i} \int_{|t|=1}^{\pi} h(t) \cdot|u(t)|^{2} \cdot t^{-1} d t= \\
& =\frac{1}{2 \pi i} \int_{-\pi}^{\pi} \widehat{h}(\Theta) \cdot|\widehat{u}(\Theta)|^{2} \cdot \exp (-i \Theta) \cdot i \cdot \exp (i \Theta) d \Theta= \\
& =\frac{1}{2 \pi} \int_{-\pi}^{\pi} \widehat{h}(\Theta) \cdot|\widehat{u}(\Theta)|^{2} d \Theta
\end{aligned}
$$

(изменение порядка интегрирования и суммирования допустимо, так как $u=\left\{u_{n}\right\}_{n=-\infty}^{\infty}-$ финитная последовательность). Таким образом, равенство

$$
(H u, u)=\frac{1}{2 \pi} \int_{-\pi}^{\pi} \widehat{h}(\Theta) \cdot|\widehat{u}(\Theta)|^{2} d \Theta
$$

справедливо для любого ядра $h \in l_{1}$ и любой финитной последовательности $u=\left\{u_{n}\right\}_{n=-\infty}^{\infty}$.

По теореме Хаусдорфа-Юнга (см. [13, п. 13.5]) имеем

$$
\|\widehat{h}\|_{L_{\infty}(-\pi, \pi)} \leqslant\|h\|_{1}, \quad\|\widehat{u}\|_{L_{2}(-\pi, \pi)} \leqslant\|u\|_{2} .
$$


Поэтому

$$
\left.\left.\left|\int_{-\pi}^{\pi} \widehat{h}(\Theta) \cdot\right| \widehat{u}(\Theta)\right|^{2} d \Theta\left|\leqslant\|\widehat{h}\|_{L_{\infty}(-\pi, \pi)} \int_{-\pi}^{\pi}\right| \widehat{u}(\Theta)\right|^{2} d \Theta \leqslant\|\widehat{h}\|_{L_{\infty}(-\pi, \pi)}\|u\|_{2}^{2}, \quad u \in l_{2} .
$$

Кроме того, на основании неравенств Коши-Буняковского и Юнга имеем

$$
|(H u, u)| \leqslant\|H u\|_{2}\|u\|_{2} \leqslant\|h\|_{1}\|u\|_{2}^{2}, \quad u \in l_{2} .
$$

Таким образом, в силу (21) и (22), обе части равенства (20) определены при любом $u \in l_{2}$, представляют собой линейные непрерывные функционалы в пространстве $l_{2}$ и совпадают при $h \in l_{1}$, если $u=\left\{u_{n}\right\}_{n=-\infty}^{\infty}-$ произвольная финитная последовательность. Поскольку множество всех финитных последовательностей плотно в пространстве $l_{2}$, то равенство (20) справедливо для любого $u \in l_{2}$. Следовательно, в силу условия (11), справедливо неравенство

$$
\operatorname{Re}(H u, u)=\frac{1}{2 \pi} \int_{-\pi}^{\pi} \operatorname{Re} \widehat{h}(\Theta) \cdot|\widehat{u}(\Theta)|^{2} d \Theta \geqslant 0, \quad u \in l_{2},
$$

равносильное неравенству (19), т.е. оператор свертки $H$ является положительным в гильбертовом пространстве $l_{2}$.

Наконец, докажем положительность оператор свертки $B$ в пространстве $l_{p}$. В самом деле, так как $B u=\bar{b} \cdot H(b \cdot u)$ и, как доказано в пп. $1-3, b \cdot u \in l_{2}$ при всех $u \in l_{p}$, то на основании равенства (15) имеем

$$
\langle B u, u\rangle=\langle\bar{b} \cdot H(b \cdot u), u\rangle=\langle H(b \cdot u), b \cdot u\rangle=(H(b \cdot u), b \cdot u)=\frac{1}{2 \pi} \int_{-\pi}^{\pi} \widehat{h}(\Theta) \cdot|\widehat{b \cdot u}(\Theta)|^{2} d \Theta, \quad u \in l_{2},
$$

откуда вытекает равенство (15) для всех $u \in l_{2}$. Из равенства (15), подобно тому как из равенства (20) было получено неравенство (23), в силу условия (11) сразу получаем неравенство $\operatorname{Re}\langle B u, u\rangle \geqslant 0, u \in l_{p}$, т.е. оператор $B$ является положительным в пространстве $l_{p}$ при любом $p \in(1, \infty)$.

\section{3. Нелинейные интегральные уравнения с разностными ядрами.}

3.1. Случай пространств Лебега. Рассмотрим в вещественном пространстве Лебега $L_{p}(0,1)$, $1 \leqslant p<\infty$, с обычной нормой

$$
\|u\|_{p}=\left[\int_{0}^{1}|u(x)|^{p} d x\right]^{1 / p}
$$

интегральный оператор

$$
\left(P_{01}^{\varphi} u\right)(x)=\int_{0}^{1} \varphi(|x-t|) \cdot u(t) d t .
$$

Определение 1. Запись $\varphi(x) \in \Omega(0,1]$ означает, что $\varphi(x)$ - такая непрерывная, невозрастающая, выпуклая вниз в промежутке $(0,1]$ функция, что

$$
\int_{0}^{1} \varphi(x) d x \geqslant 0 .
$$

Лемма 5 (см. [3]). Пусть $1<p<\infty$ и ядро $\varphi(x)$ удовлетворяет условию

$$
\begin{array}{ll}
\varphi(x) \in L_{p^{\prime} / 2}(0,1) \cap \Omega(0,1], & \text { если } 1<p \leqslant 2, \\
\varphi(x) \in L_{1}(0,1) \cap \Omega(0,1], & \text { если } 2<p<\infty .
\end{array}
$$


Тогда оператор $P_{01}^{\varphi}$ действует непрерывно из пространства $L_{p}(0,1)$ в сопряженное с ним пространство $L_{p^{\prime}}(0,1), p^{\prime}=p /(p-1)$, и положителен, причем для любой функиии $u(x) \in L_{p}(0,1)$ справедливы следующие неравенства:

$$
\begin{gathered}
\left\|P_{01}^{\varphi} u\right\|_{p^{\prime}} \leqslant c(\varphi)\|u\|_{p} \\
\left\langle P_{01}^{\varphi} u, u\right\rangle=\frac{a_{0}}{2}\left[\int_{0}^{1} u(x) d x\right]^{2}+\sum_{n=1}^{\infty} a_{n}\left|\int_{0}^{1} u(x) e^{i \pi n x} d x\right|^{2} \geqslant 0,
\end{gathered}
$$

где

$$
\begin{gathered}
a_{n}=2 \int_{0}^{1} \varphi(x) \cos (\pi n x) d x \geqslant 0, \quad n=0,1,2, \ldots, \\
c(\varphi)= \begin{cases}2^{2 / p^{\prime}\|\varphi\|_{p^{\prime} / 2},} & \text { если } 1<p \leqslant 2, \\
2\|\varphi\|_{1}, & \text { если } 2<p<\infty .\end{cases}
\end{gathered}
$$

Замечание 1. Поскольку функции $\varphi(x)=x^{\alpha-1}$ при $0<\alpha<1$ и $\varphi(x)=-\ln x$ принадлежат классу $\Omega(0,1]$, то лемма 5 охватывает потенциал Рисса и логарифмический потенциал (см. [11]).

В [1, § 15], [3] доказаны теоремы о существовании, единственности и оценках решений для различных классов нелинейных уравнений, содержащих оператор $P_{01}^{\varphi}$, в пространствах Лебега. Покажем, что в случае нечетностепенных нелинейностей эти решения могут быть найдены градиентным методом.

Теорема 4. Пусть $p>2$ - четное число и $\varphi(x) \in L_{1}(0,1) \cap \Omega(0,1]$. Тогда для любого $f(x) \in$ $L_{p^{\prime}}(0,1)$ уравнение

$$
u^{p-1}(x)+\int_{0}^{1} \varphi(|x-t|) u(t) d t=f(x)
$$

имеет единственное решение $u^{*}(x) \in L_{p}(0,1)$. Это решение можно найти методом последовательных приближений по формуле

$$
u_{n+1}=u_{n}-\delta_{n} \cdot\left\|B u_{n}-f\right\|_{p^{\prime}}^{2-p^{\prime}}\left|B u_{n}-f\right|^{p^{\prime}-2}\left(B u_{n}-f\right),
$$

где $n=0,1,2,3, \ldots, u_{0}(x) \in L_{p}(0,1)$ - начальное приближение, $B u=u^{p-1}+P_{01}^{\varphi} u$,

$$
\delta_{n}=\min \left(1, \frac{2}{\varepsilon+(p-1)\left(\left\|u_{n}\right\|_{p}+\left\|B u_{n}-f\right\|_{p^{\prime}}\right)^{p-2}+2\|\varphi\|_{1}}\right), \quad \varepsilon>0 .
$$

Доказательство. Запишем уравнение (27) в операторном виде:

$$
A u=f, \quad \text { где } \quad A u=u^{p-1}+P_{01}^{\varphi} u .
$$

Поскольку для любой функции $u(x) \in L_{p}(0,1)$ имеем $u^{p-1}(x) \in L_{p^{\prime}}(0,1)$, то в силу леммы 5 оператор $A$ действует из $L_{p}(0,1)$ в сопряженное с ним пространство $L_{p^{\prime}}(0,1)$. Так как $p$ четное число и оператор $P_{01}^{\varphi}$ положителен, то оператор $A$ является строго монотонным и коэрцитивным. Значит, по теореме 1 уравнение $(27)$ имеет единственное решение $u^{*}(x) \in L_{p}(0,1)$. Покажем, что это решение можно найти по формуле $(28)$. Для этого заметим, что пространство $L_{p}(0,1)$ при $1<p<\infty$ является строго выпуклым, и дуализующее отображение $J^{*}$ для пространства $L_{p^{\prime}}(0,1)$ имеет вид

$$
\left(J^{*} w\right)(x)=\|w\|_{p^{\prime}}^{2-p^{\prime}}|w(x)|^{p^{\prime}-2} w(x) .
$$

В силу интегрального неравенства Минковского для всех $u(x), v(x) \in L_{p}(0,1)$ имеем

$$
\|A u-A v\|_{p^{\prime}} \leqslant\left\|u^{p-1}-v^{p-1}\right\|_{p^{\prime}}+\left\|P_{01}^{\varphi}(u-v)\right\|_{p^{\prime}}=I_{1}+I_{2} .
$$

Оценим $I_{1}$. Так как

$$
\left|t^{p-1}-s^{p-1}\right| \leqslant \frac{p-1}{2}|t-s|\left(t^{p-2}+s^{p-2}\right)
$$


для любых $t, s \in \mathbb{R}$, то

$$
\begin{gathered}
I_{1}=\left\|u^{p-1}-v^{p-1}\right\|_{p^{\prime}} \leqslant \frac{p-1}{2}\left[\int_{a}^{b}|u(x)-v(x)|^{p^{\prime}}\left|u^{p-2}(x)+v^{p-2}(x)\right|^{p^{\prime}} d x\right]^{1 / p^{\prime}} \leqslant \\
\leqslant \frac{p-1}{2}\|u-v\|_{p}\left(\|u\|_{p}^{p-2}+\|v\|_{p}^{p-2}\right) \leqslant(p-1) R^{p-2}\|u-v\|_{p},
\end{gathered}
$$

где $R=\max \left(\|u\|_{p},\|v\|_{p}\right)$; здесь мы сначала применили неравенство Гельдера с показателями $p / p^{\prime}$ и $p /\left(p-p^{\prime}\right)$, а затем ко второму сомножителю применили неравенство Минковского. Оценка $I_{2}$ содержится в лемме 5 .

Таким образом, с учетом леммы 5 имеем

$$
\|A u-A v\|_{p^{\prime}} \leqslant \mu(R) \cdot\|u-v\|_{p},
$$

где $\mu(R)=(p-1) R^{p-2}+2\|\varphi\|_{1}$ - возрастающая на $[0, \infty)$ функция. Значит, $A$ - ограниченный липшиц-непрерывный оператор.

Далее, используя положительность оператора $P_{01}^{\varphi}$ и неравенство $\left(t^{p-1}-s^{p-1}\right)(t-s) \geqslant 2^{2-p}|t-s|^{p}$, справедливое для всех $t, s \in \mathbb{R}$, имеем

$$
\begin{aligned}
\langle A u-A v, u-v\rangle \geqslant \int_{a}^{b}\left[u^{p-1}(x)-v^{p-1}(x)\right][u(x)-v(x)] d x \geqslant & \\
& \geqslant 2^{2-p} \int_{a}^{b}|u(x)-v(x)|^{p} d x=\beta\left(\|u-v\|_{p}\right),
\end{aligned}
$$

где $\beta(s)=2^{2-p} s^{p}$ - такая строго возрастающая на $[0, \infty)$ функция, что $\beta(0)=0$, т.е. $A$ - равномерно монотонный оператор.

Так как $\left\|u^{p-1}\right\|_{p^{\prime}}=\|u\|_{p}^{p-1}$, то оператор $F u=u^{p-1}$ действует непрерывно из $L_{p}(0,1)$ в $L_{p^{\prime}}(0,1)$ и в силу [8, Лемма 4.1] является потенциальным. В свою очередь, оператор $P_{01}^{\varphi}$ с четным ядром $\varphi(x)$ является симметрическим и поэтому (см. Пример 1) также является потенциальным. Таким образом, оператор $A$ как сумма двух непрерывных потенциальных операторов является непрерывным потенциальным оператором, действующим из $L_{p}(0,1)$ в $L_{p^{\prime}}(0,1)$. Значит, на основании теоремы 1 , последовательность $(28)$ сходится к $u^{*}(x)$ по норме пространства $L_{p}(0,1)$, что и требовалось доказать.

3.2. Случай весовых пространств Лебега. Пусть $\rho(x)$ есть неотрицательная почти всюду конечная и почти всюду отличная от нуля измеримая на $[0,1]$ функция. Обозначим через $L_{01}^{p}(\rho)$, $p \geqslant 1$, множество всех измеримых на отрезке $[0,1]$ функций $u(x)$ с конечной нормой

$$
\|u\|_{p, 1}=\left[\int_{0}^{1} \rho(x) \cdot|u(x)|^{p} d x\right]^{1 / p} .
$$

Известно (см., например, [7]), что $L_{01}^{p}(\rho)$ при $1<p<\infty$ есть строго выпуклое рефлексивное банахово пространство и сопряженным с ним является пространство $L_{01}^{p^{\prime}}\left(\rho^{1-p^{\prime}}\right)$, где $p^{\prime}=p /(p-1)$. Если $\rho(x)=1$, то будем писать $L_{01}^{p}$ и $\|\cdot\|_{p}$. Норму в $L_{01}^{p^{\prime}}\left(\rho^{1-p^{\prime}}\right)$ будем обозначать через $\|\cdot\|_{p^{\prime}, 1-p^{\prime}}$.

Аналогично теореме 3 доказывается следующее утверждение.

Теорема 5. Пусть $p \geqslant 4$-четное число, ядро $\varphi(x) \in L_{01}^{1} \cap \Omega(0,1]$ и выполнено условие

$$
c(\rho)=\left[\int_{0}^{1}[\rho(x)]^{2 /(2-p)} d x\right]^{(p-2) /(2 p)}<\infty .
$$


Тогда для всех $f(x) \in L_{01}^{p^{\prime}}\left(\rho^{1-p^{\prime}}\right)$ уравнение

$$
\rho(x) \cdot u^{p-1}(x)+\int_{0}^{1} \varphi(|x-t|) u(t) d t=f(x)
$$

имеет единственное решение $u^{*}(x) \in L_{01}^{p}(\rho)$. Это решение можно найти методом последовательных приближений по формуле

$$
u_{n+1}=u_{n}-\delta_{n} \cdot\left\|B u_{n}-f\right\|_{p^{\prime}, 1-p^{\prime}}^{2-p^{\prime}} \varrho^{1-p^{\prime}}\left|B u_{n}-f\right|^{p^{\prime}-2}\left(B u_{n}-f\right),
$$

где $n=0,1,2,3, \ldots, u_{0}(x) \in L_{01}^{p}(\rho)$ - начальное приближение, $B u=\varrho \cdot u^{p-1}+P_{01}^{\varphi} u$,

$$
\delta_{n}=\min \left(1, \frac{2}{\varepsilon+(p-1)\left(\left\|u_{n}\right\|_{p, 1}+\left\|B u_{n}-f\right\|_{p^{\prime}, 1-p^{\prime}}\right)^{p-2}+2 c^{2}(\rho)\|\varphi\|_{1}}\right), \quad \varepsilon>0 .
$$

В заключение отметим, что при исследовании методом монотонных (по Браудеру-Минти) операторов нелинейных дискретных и интегральных уравнений с разностными ядрами важную роль играет свойство положительности (по Бохнеру) соответствующих дискретных и интегральных операторов. Условия положительности для некоторых классов таких операторов приведены, например, в $[1,5,11]$.

\section{СПИСОК ЛИТЕРАТУРЫ}

1. Асхабов C. Н. Нелинейные уравнения типа свертки. - М.: Физматлит, 2009.

2. Асхабов $C$. Н. Приближенное решение нелинейных дискретных уравнений типа свертки// Совр. мат. Фундам. напр. - 2012. - 45. - С. 18-31.

3. Асхабов С. Н. Нелинейные уравнения типа свертки в пространствах Лебега// Мат. заметки. - 2015. -97 , № 5. - C. $643-654$.

4. Асхабов C. Н. Нелинейные интегральные уравнения с ядрами типа потенциала на отрезке// Совр. мат. Фундам. напр. - 2016. - 60. - С. 5-22.

5. Асхабов С. Н. Условия положительности операторов с разностными ядрами в рефлексивных пространствах// Итоги науки и техн. Сер. Совр. мат. и ее прил. Темат. обз. - 2018. - 149. - С. 3-13.

6. Асхабов С. Н., Джабраилов А. Л. Приближенное решение нелинейных уравнений типа свертки на отрезке// Уфим. мат. ж. - 2013. - 5, № 2. - С. 3-11.

7. Вайнберг M. M. Вариационный метод и метод монотонных операторов в теории нелинейных уравнений. - М.: Наука, 1972.

8. Гаевский X., Грегер K., Захариас K. Нелинейные операторные уравнения и операторные дифференциальные уравнения. - М.: Мир, 1978.

9. Гахов Ф. Д., Черский Ю. И. Уравнения типа свертки. - М.: Наука, 1978.

10. Дедагич Ф., Забрейко П. П. Об операторах суперпозиции в пространствах $l_{p} / /$ Сиб. мат. ж. - 1987. - 28, № 1. - С. 86- 98 .

11. Нахущев А. М. Дробное исчисление и его применение. - М.: Физматлит, 2003.

12. Эдвардс Р. Ряды Фурье в современном изложении. Т. 1. - М.: Мир, 1985.

13. Эдвардс Р. Ряды Фурье в современном изложении. Т. 2. - М.: Мир, 1985.

14. Dedagic F., Halilovic S., Barakovic E. On the solvability of discrete nonlinear Hammerstein systems in spaces// Math. Balkan. New Ser. - 2012. — 26, № 3--4. - P. 325--333.

Асхабов Султан Нажмудинович

Чеченский государственный педагогический университет, Грозный;

Чеченский государственный университет, Грозный

E-mail: askhabov@yandex.ru 